\title{
PST thin films for electrocaloric coolers
}

T. M. Correia ${ }^{1, a)}$, S. Kar-Narayan ${ }^{2}$, J. S. Young ${ }^{2}$, J. F. Scott ${ }^{3}$, N. D. Mathur ${ }^{2}$, R. W. Whatmore ${ }^{4}$ and Q. Zhang ${ }^{1, b)}$

${ }^{1}$ Department of Materials, Cranfield University

Bedfordshire MK43 OAL, United Kingdom

${ }^{2}$ Department of Materials Science, University of Cambridge, Pembroke Street, Cambridge CB2 3QZ, United Kingdom

${ }^{3}$ Cavendish Laboratory, Department of Physics, University of Cambridge, Cambridge CB3 0HE, United Kingdom

${ }^{4}$ Tyndall National Institute, University College Cork, Lee Maltings, Dyke Parade, Cork, Ireland

\begin{abstract}
Relaxor behaviour in a thin film of partially ordered $\mathrm{PbSc}_{0.5} \mathrm{Ta}_{0.5} \mathrm{O}_{3}$ (PST) was confirmed via slim $P-E$ loops and the frequency dependence of the temperature at which the dielectric constant is maximum. Indirect measurements of the electrocaloric effect suggest that removing a field of $774 \mathrm{kV} \mathrm{cm}^{-1}$ yields a temperature change of $-3.5^{\circ} \mathrm{C}$ to $-6.9^{\circ} \mathrm{C}$ over a broad range of operating temperatures near room temperature $\left(1{ }^{\circ} \mathrm{C}-127^{\circ} \mathrm{C}\right)$, with a correspondingly large refrigerant capacity of $662 \mathrm{~J} \mathrm{~kg}^{-1}$. In addition to low electrical hysteresis, there is negligible thermal hysteresis. PST thin films are therefore promising for EC cooling near room temperature.
\end{abstract}

\section{Introduction}

Electrocaloric (EC) coolers have stimulated great attention since the recent discovery of the giant EC effect in ferroelectric thin films [1-2]. This has sparked the desire to develop an environmentally friendly, powerful and low-cost EC refrigerator [3-7]. The EC effect is typically characterized by the entropy change, $\Delta S$, driven by the application/removal of an electric field, $E$, associated with a temperature change, $\Delta T$ as follows:

\footnotetext{
a) tatiana.correia@npl.co.uk

Current address: National Physical Laboratory, Hampton Road, Teddington, Middlesex, TW11 0LW

b) q.zhang@cranfield.ac.uk
} 


$$
\Delta S=-\frac{1}{\rho} \int_{E_{1}}^{\Sigma_{2}}\left(\frac{\partial P}{\partial T}\right) d E
$$

and,

$$
\Delta T=-\frac{1}{\rho} \int_{E_{1}}^{L_{2}} \frac{T}{c}\left(\frac{\partial P}{\partial T}\right) d E
$$

where $P$ is polarization, $c$ is specific heat capacity, $\rho$ is density and $E_{1}$ and $E_{2}$ are the starting and final applied fields, respectively. These equations are not straightforward to apply if there is electrical or thermal hysteresis, e.g. near first-order phase transitions or within the "glassy" nonergodic state of some relaxors [5].

Lead scandium tantalate, $\mathrm{PbSc}_{0.5} \mathrm{Ta}_{0.5} \mathrm{O}_{3}$ (PST), has a perovskite structure, $A\left(B^{\prime} B^{\prime \prime}\right) \mathrm{O}_{3}$, in which $B$-site disorder leads to relaxor behaviour. Appropriate heat treatments promote $B$-site ordering, and so long-range ferroelectric order may be observed [8-10]. Relaxor PST undergoes a diffuse second-order phase transition whereas a first-order phase transition is found in highly ordered PST [8-10]. While a paraelectric (or relaxor)-ferroelectric phase transition is known to occur between $0{ }^{\circ} \mathrm{C}$ and room temperature depending on the degree of PST ordering $[8,10]$, dielectric measurements of PST thin films have indicated the existence of several anomalies at cryogenic temperatures [11]. The underlying mechanisms are unresolved but could involve structural phase transitions and domain-wall dynamics. Here we investigate the EC effect in a partially ordered $\mathrm{PbSc}_{0.5} \mathrm{Ta}_{0.5} \mathrm{O}_{3}$ thin film, which is expected to exhibit a high pyroelectric coefficient, a phase transition near room temperature and slim relaxor-like $P-E$ loops [12-15]. Furthermore, PST compositions with $\Omega<0.82$ [8] have demonstrated a second-order phase transition and so no thermal hysteresis is expected.

\section{Experimental}

A partially ordered $\mathrm{PbSc}_{0.5} \mathrm{Ta}_{0.5} \mathrm{O}_{3}$ thin film was fabricated by sol-gel using precursors of lead acetate trihydrate $\mathrm{Pb}(\mathrm{OAc})_{2} \cdot 3 \mathrm{H}_{2} \mathrm{O}$, scandium acetate $\left(\mathrm{Sc}(\mathrm{OAc})_{3}\right.$, and tantalum ethoxide $\mathrm{Ta}(\mathrm{OEt})_{5}$. The scandium acetate and tantalum ethoxide were mixed together, dissolved in 2methoxyethanol (2ME) and refluxed for $10 \mathrm{hrs}$. Separately, lead acetate trihydrate was dissolved in $2 \mathrm{ME}$, and the water was distilled out of the system. Both solutions were mixed and stirred at room temperature for $2 \mathrm{hrs}$. The final concentration of the synthesized PST sol was 0.3M. PST sol was spin-coated at $3000 \mathrm{rpm}$ for $30 \mathrm{sec}$ onto a $\mathrm{Pt} / \mathrm{Ti} / \mathrm{SiO}_{2} / \mathrm{Si}$ substrate. Each layer was 
pyrolized at $350{ }^{\circ} \mathrm{C}$ for 1 min. After the deposition of 5 layers, the film was annealed at $650{ }^{\circ} \mathrm{C}$ via Rapid Thermal Annealing with a heating ramp of $18^{\circ} \mathrm{C} / \mathrm{sec}$. The thickness of each layer was $20 \mathrm{~nm}$, and the above-mentioned process was repeated ten times to obtain the desired $200 \mathrm{~nm}$. $350 \times 350 \mu \mathrm{m}^{2}$ top electrodes of $\mathrm{Cr} / \mathrm{Au}$ were deposited by thermal evaporation (Fig. 1). Dielectric constant measurements were carried out using a Wayne-Kerr impedance analyzer (Wayne Kerr Electronics, UK). $P-E$ loops were obtained by means of a Radiant Technologies RT66A ferroelectric tester (Radiant Technologies Inc., USA). A Peltier element was used for temperature control.

\section{Results and Discussion}

X-Ray diffraction data (Fig. 2) suggest that the film adopts a single-phase perovskite structure and shows preferential $\{111\}$ orientations. The degree of $B$-site chemical order was found to be $\Omega=0.32$ from the relative intensities $I$ of the $1 / 21 / 21 / 2$ and 100 superlattice reflections, according to [16]:

$$
\Omega=\frac{\left(I_{\frac{11 \frac{1}{2}}{2}} / I_{100}\right)_{\text {observed }}}{\left(I_{\frac{11 \frac{1}{2}}{2}} / I_{100}\right)_{\text {calculated } \Omega=1}} .
$$

The temperature dependences of the dielectric constant, $\varepsilon$, and loss tangent, $\tan \delta$, at different frequencies are illustrated in Fig. 3. Dielectric constant and loss tangent show maxima at $T_{\mathrm{m}}$ near room temperature, and the strong frequency dependence indicates a relaxorferroelectric phase transition. The similarity in $|\mathrm{d} \varepsilon / \mathrm{d} T|$ below and above $T_{\mathrm{m}}$ confirms a secondorder phase transition. The values of $\varepsilon$ in our PST thin film are smaller than those in previously reported partially ordered PST thin films (between 1000-6000) which were annealed at $T>$ $700{ }^{\circ} \mathrm{C}[10]$. This may be due to the use of a lower annealing temperature here $\left(650{ }^{\circ} \mathrm{C}\right)$, resulting in a smaller average grain size.

In order to evaluate the EC effect in the PST thin film, $P-E$ loops at $1 \mathrm{kHz}$ were measured at different temperatures, as shown in Fig. 4. In addition to the frequency dependence of $T_{\mathrm{m}}$ (Fig. 3), the slim $P-E$ loops corroborate previously reported relaxor behaviour of partially ordered PST thin films [10]. The temperature dependence of the polarization at different fields, established from the upper branches of the hysteresis loops in $E>0$ upon cooling and heating, is presented in Fig. 5. The PST thin film exhibits negligible thermal hysteresis. Given that we are 
in an ergodic regime, unlike the relaxor polymer poly(vinylidene fluoridetrifluoroethylenechlorofluoroethylene [P(VDF-TrFE-CFE)] for which direct and indirect measurements do not match [17], we may apply the indirect thermodynamic analysis to evaluate EC performance. Using Equations (1) and (2), with $c=0.32 \mathrm{~J} \mathrm{~K}^{-1} \mathrm{~g}^{-1}$ and $\rho=8.8 \mathrm{~g} \mathrm{~cm}^{-3}$ [18], we determined $\Delta S$ vs $T$ and $\Delta T$ vs $T$ for different $\Delta E$ using the cooling data (Figs $5 \mathrm{a} \& \mathrm{~b}$, respectively). There is evidence for peak in both $\Delta S=-6.3 \mathrm{~J} \mathrm{~K}^{-1} \mathrm{~kg}^{-1}$ and $\Delta T=-6.2{ }^{\circ} \mathrm{C}$ at $68{ }^{\circ} \mathrm{C}$, probably due to a fieldinduced relaxor-ferroelectric phase transition. In our PST thin film, $|\Delta S|=4.5-6.3 \mathrm{~J} \mathrm{~K}^{-1} \mathrm{~kg}^{-1}$ which does not exceed the values for e.g. $\mathrm{PbZr}_{0.95} \mathrm{Ti}_{0.05} \mathrm{O}_{3}$ and $\mathrm{P}(\mathrm{VDF}-\mathrm{TrFE})$ 55:45 mol \% copolymers ( $8 \mathrm{~J} \mathrm{~K}^{-1} \mathrm{~kg}^{-1}$ and $60 \mathrm{~J} \mathrm{~K}^{-1} \mathrm{~kg}^{-1}$, respectively) [1], as expected given that we are working near a second-order phase transition. However, a broad temperature range near room temperature may be exploited.

It has been suggested $[19,20]$ that one may compare magnetocaloric materials by evaluating refrigerant capacity $(R C)$, and by extension EC materials. From [19] we have:

$$
R C=\int_{T_{1}}^{T_{2}} \Delta S(T) d T,
$$

where $T_{1}$ and $T_{2}$ are the cold and hot temperatures accessed via the EC effect. Within the temperature range studied, $\Delta S$ varies by less than $27 \%$ (Fig. 6a) and contributes significantly to $R C$. We estimate $R C=695 \mathrm{~J} \mathrm{~kg}^{-1}$ in the limits $T_{1}=1^{\circ} \mathrm{C}$ and $T_{2}=127^{\circ} \mathrm{C}$ for $\Delta E=774 \mathrm{kV} \mathrm{cm}^{-1}$. The maximum hysteresis loss, determined from $P-E$ loop area for $E>0$ at $9^{\circ} \mathrm{C}$, could at most reduce the $R C$ by just $4.8 \%$ to $662 \mathrm{~J} \mathrm{~kg}^{-1}$. Hence the $R C$ we predict from Equation (1) is a reasonable estimate for our relaxor films. The fact that, the estimated RC value in PST thin film is much higher than that in magnetocaloric Gd-based materials $\left(360 \mathrm{~J} \mathrm{~kg}^{-1}\right)$ [20], indicates that electrocaloric PST thin films may be used as a media in a cost-efficient refrigeration system operating near room temperature.

\section{Conclusions}

A relaxor thin film of $\mathrm{PbSc}_{0.5} \mathrm{Ta}_{0.5} \mathrm{O}_{3}$ with partial $B$-site order $(\Omega=0.32)$ was fabricated by solgel, and subjected to rapid thermal annealing. Its relaxor nature near room temperature was confirmed by slim loops and the frequency dependence of $T_{\mathrm{m}}$. PST thin films could be promising for EC refrigeration, as they combine a set of remarkable properties: 1) a large EC effect below and above room temperature of $\Delta S \sim-5 \mathrm{~J} \mathrm{~K}^{-1} \mathrm{~kg}^{-1}$ for under $774 \mathrm{kV} \mathrm{cm}^{-1}$, 2) no thermal 
hysteresis, 3) low electrical losses of $4.8 \%$ and 4) a small variation of $\Delta S$ within $1{ }^{\circ} \mathrm{C}-127{ }^{\circ} \mathrm{C}$ which yields a large $R C=662 \mathrm{~J} \mathrm{~kg}^{-1}$ for $\Delta E=774 \mathrm{kV} \mathrm{cm}^{-1}$.

\section{Acknowledgements}

The authors thank the Engineering and Physical Sciences Research Council (EPSRC) for the financial support granted to this work under contract EP/E035043 (Cranfield) and EP/E03389X (Cambridge).

\section{References}

[1] A. S. Mischenko, Q. Zhang, J. F. Scott, R. W. Whatmore, and N. D. Mathur, Science 311, 1270 (2006)

[2] A. S. Mischenko, Q. Zhang, R. W. Whatmore, J. F. Scott, and N. D. Mathur, Appl. Phys. Lett. 89, 242912 (2006)

[3] B. Neese, B. Chu, S.-G. Lu, Y. Wang, E. Furman, Q. M. Zhang, Science 321, 821 (2008)

[4] H. Chen, T. L. Rent, X. M. Wu, Y. Yang, L. T. Liu, Appl. Phys. Lett. 94, 182902 (2009)

[5] T. M. Correia, J. S. Young, R. W. Whatmore, J. F. Scott, N. D. Mathur, and Q. Zhang, Appl. Phys. Lett. 95, 182904 (2009)

[6] P. F. Liu, J. L. Wang, X. J. Meng, J. Yang, B. Dkhil, J. H. Chu, Huge electrocaloric effect in Langmuir-Blodgett ferroelectric polymer thin films, New J. Phys., Vol. 12: 023035-023043, 2010.

[7] H. Chen, T. L. Rent, X. M. Wu, Y. Yang, L. T. Liu, Giant electrocaloric effect in leadfree thin film of strontium bismuth tantalite, Appl. Phys. Lett., Vol. 94: 182902/1-3, 2009.

[8] C. Stenger and A. Burggraaf, Phys. Status Solidi A 61, 275 (1980)

[9] N. Setter and E. Cross, J. Appl. Phys. 51, 4356 (1980)

[10] K. Brinkman, Y. Wang, D. Su, A. Tagantsev, P. Muralt, and N. Setter, J. Appl. Phys. 102, 044110 (2007) 
[11] M. Dawber, S. Rios, J. F. Scott, Q. Zhang and R. W. Whatmore, AIP Conf. Proc. 582, 1 (2001)

[12] N. M. Shorrocks, R. W. Whatmore, P. C. Osbond, Ferroelectrics 106, 387 (1990)

[13] T. Takeishi, R.W. Whatmore, Ferroelectrics 228, 53 (1999)

[14] A. Patel, R. W. Whatmore, GB Patent 9025547 (1992)

[15] R. Watton, M. A. Todd, Ferroelectrics 118, 279 (1991)

[16] J. R. Giniewicz, A. S. Bhalla, L. E. Cross , Ferroelectrics 21, 281 (1998)

[17] S. G. Lu, B. Rožič, Q. M. Zhang, Z. Kutnjak, R. Pirc, M. Lin, X. Li, L. Gorny, Appl. Phys. Lett. 97, 202901 (2010)

[18] Y. V. Sinyavsky, V. M. Brodyansky, Experimental testing of electrocaloric cooling with transparent ferroelectric ceramic as a working body, Ferroelectrics 131, 321 (1992)

[19] K. A. Gschneidner Jr, V. K. Pecharsky and A. O. Tsokol1, Rep. Prog. Phys. 681479 (2005)

[20] V. Provenzano, A. J. Shapiro, R. D. Shull, Nature 429, 853 (2004) 


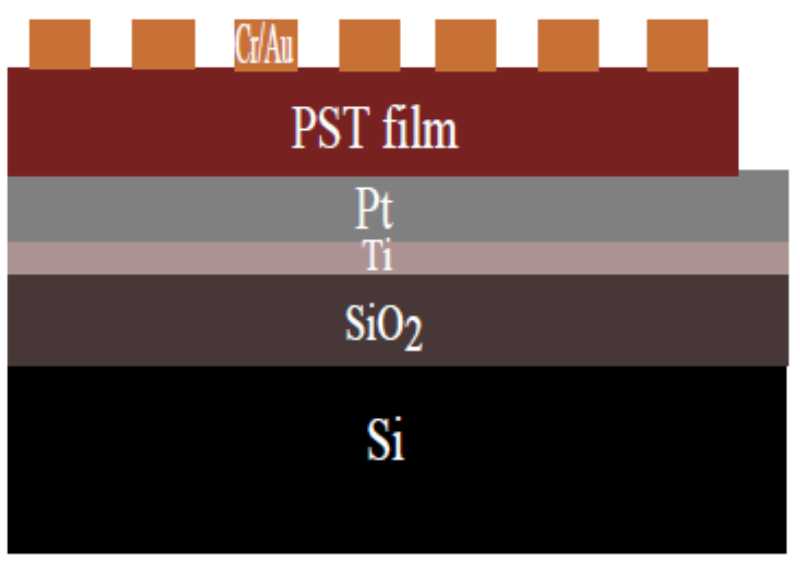

Fig. 1 Schematic of the PST thin film structure.

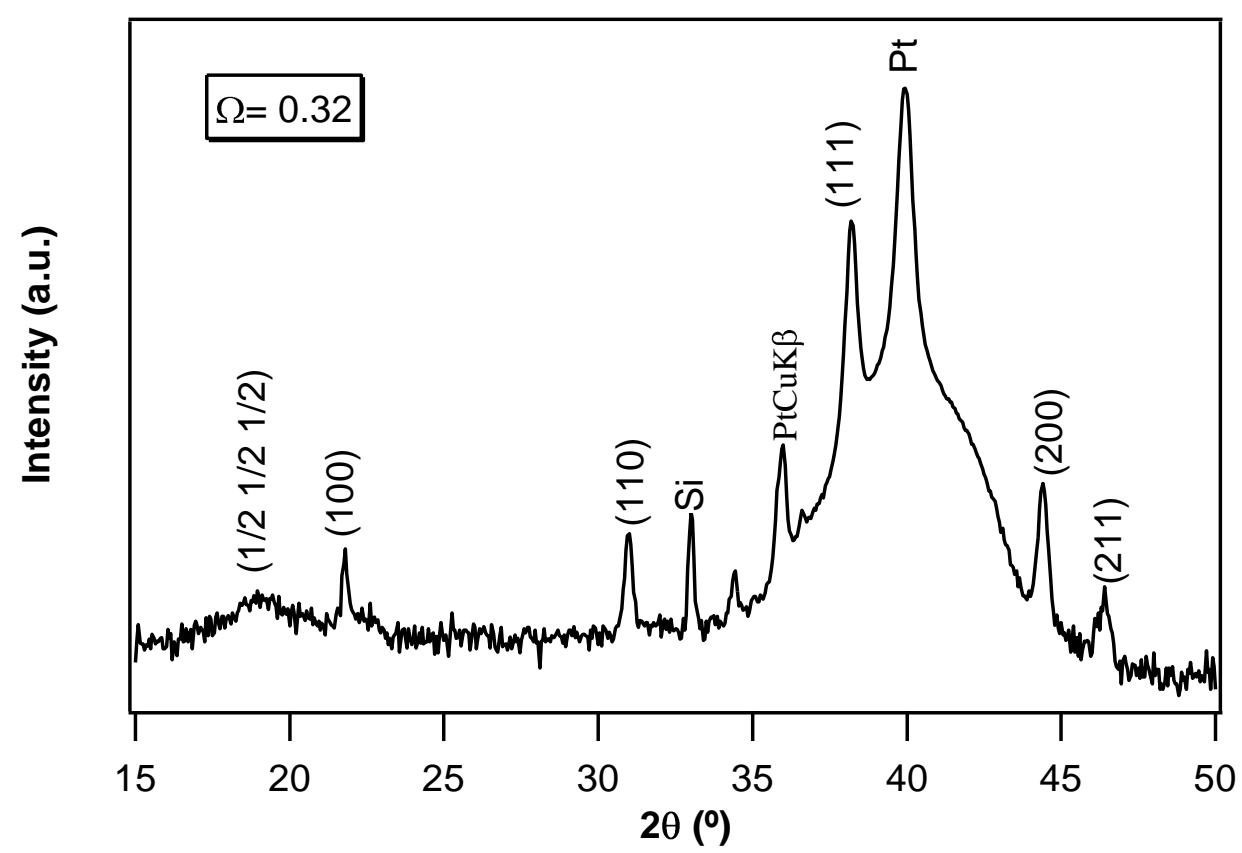

Fig. 2. XRD patterns for the PST thin film. 


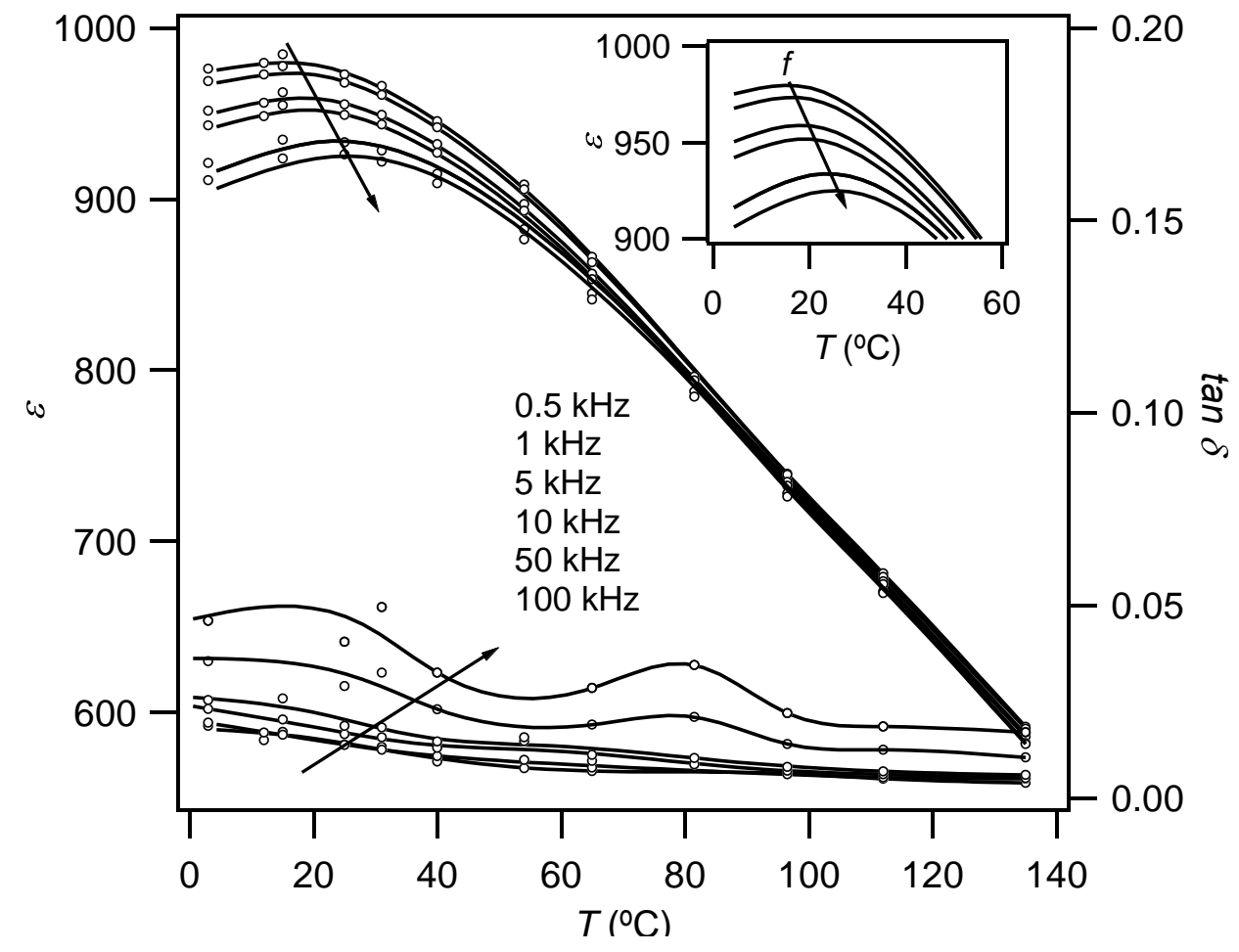

Fig. 3. Temperature dependence of dielectric constant $\varepsilon$ (upper curves) and loss tan $\delta$ (lower curves) at different frequencies. Dashed lines represent cubic-spline interpolation of raw data. 

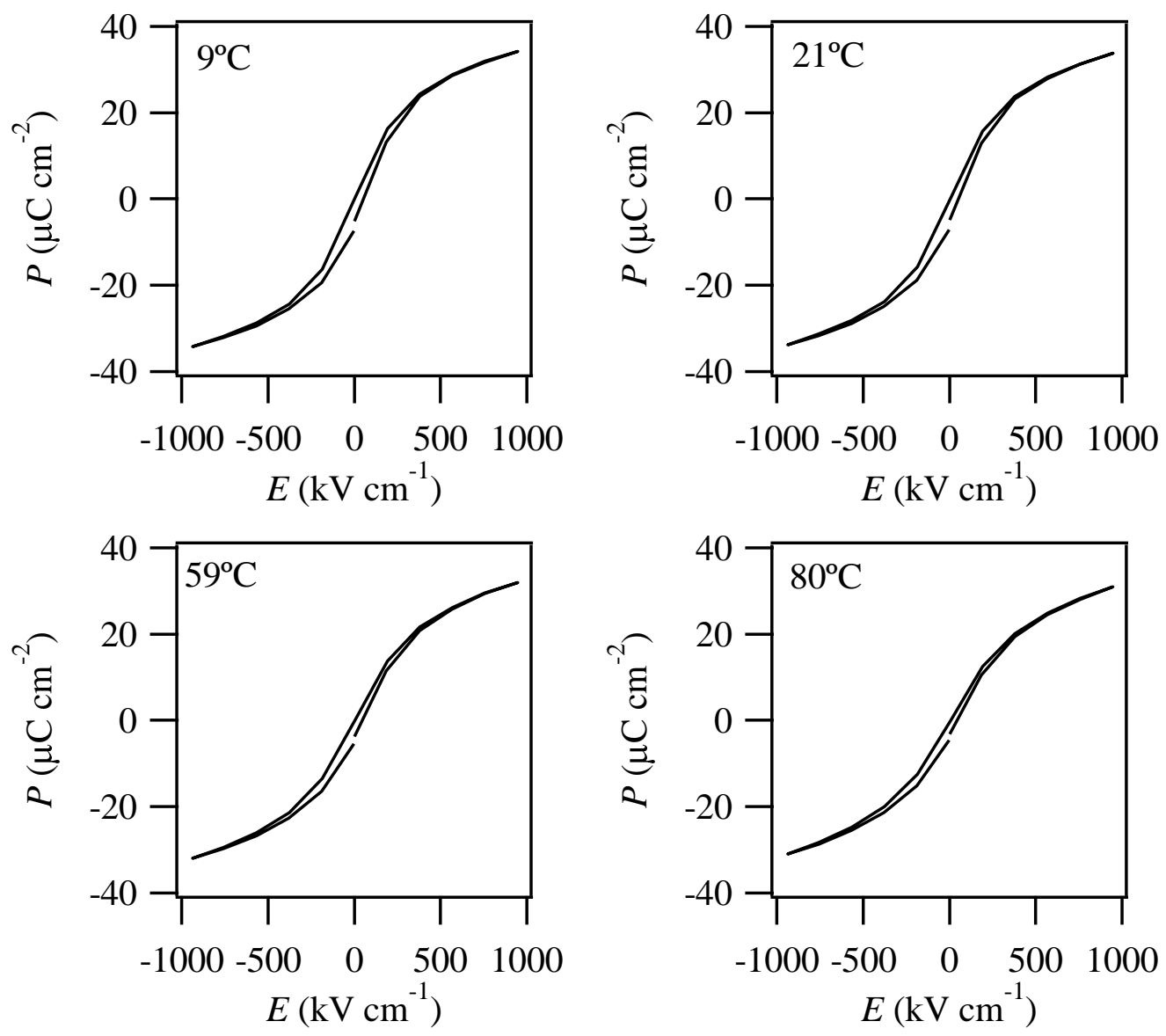

Fig. 4. $P-E$ loops at selected temperatures measured upon cooling. 


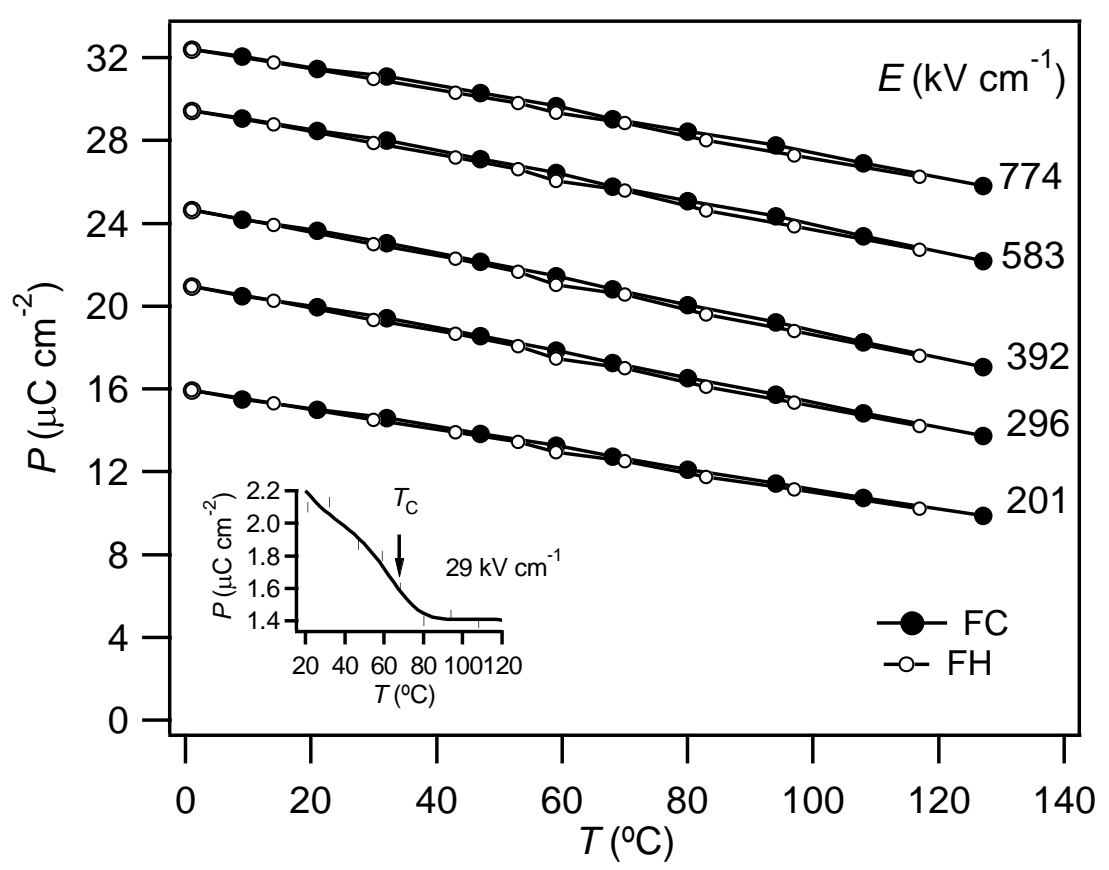

Fig. 5. Polarization as a function of temperature at selected electric field values, as deduced from the depolarizing branch of $P$-E loops $(E>0)$ taken on cooling and heating. The inset shows the temperature evolution of polarization at low field $\left(29 \mathrm{kV} \mathrm{cm}^{-1}\right)$ in order to identify the fieldinduced relaxor-ferroelectric phase transition temperature $\left(T_{\mathrm{C}}=68^{\circ} \mathrm{C}\right)$. 

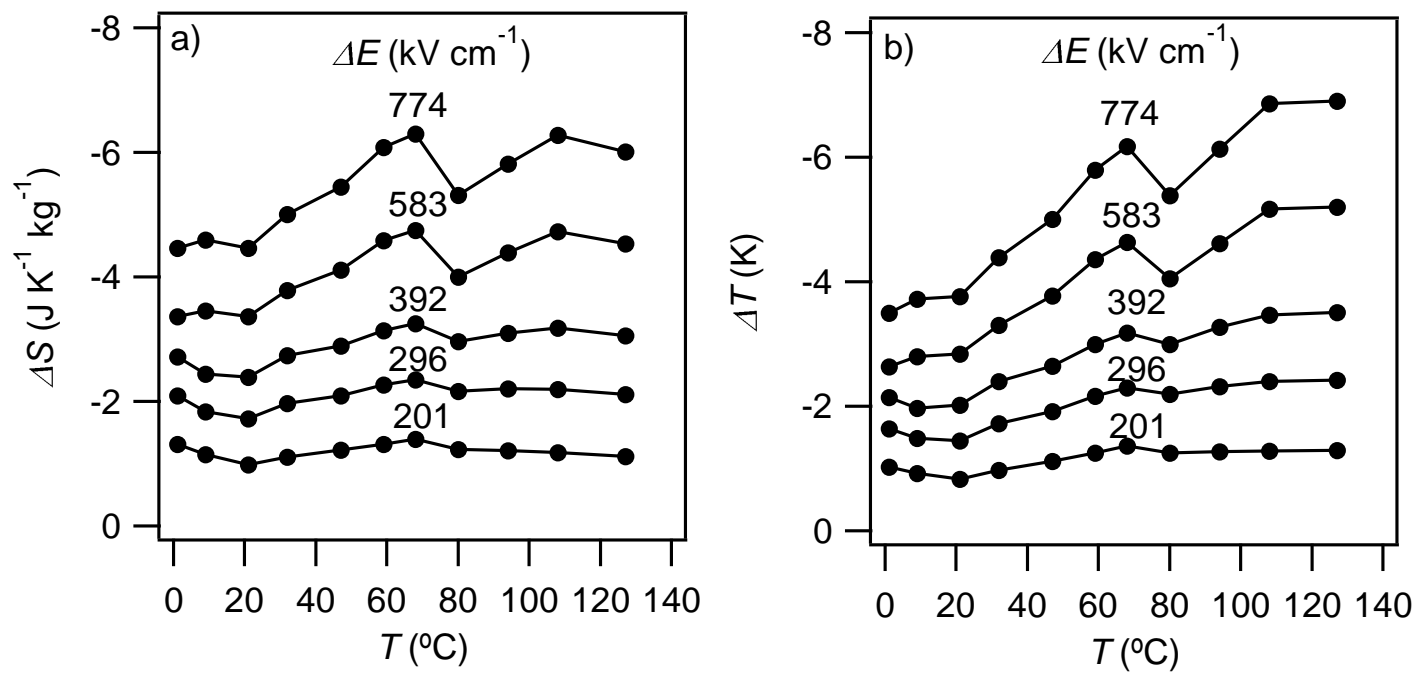

Fig. 6. EC (a) entropy change, $\Delta S$, and (b) temperature change, $\Delta T$, as a function of starting temperature, for different $\Delta E\left(E_{1}=0\right)$, predicted using Equations 1 and 2 respectively with the averaged cooling data shown in Fig. 5. 
NPL Management Ltd - Internal 